\title{
Pathologic Evaluation of Routine Nasopharynx Punch Biopsy in the Adult Population: Is It Really Necessary?
}

\author{
Sami Bercin ${ }^{1} \cdot$ Gokhan Yalciner $^{2} \cdot$ Togay Muderris $^{2} \cdot$ Fatih Gul $^{3} \cdot$ H. Mervan Deger ${ }^{4} \cdot$ Muzaffer Kiris $^{1}$ \\ ${ }^{I}$ Department of Otorhinolaryngology, Head and Neck Surgery, Ylldirtm Beyazıt University School of Medicine, Ankara; \\ ${ }^{2}$ Department of Otorhinolaryngology, Head and Neck Surgery, Ataturk Training and Research Hospital, Ankara; \\ ${ }^{3}$ Department of Otorhinolaryngology, Head and Neck Surgery, Bitlis Tatvan State Hospital, Bitlis; \\ ${ }^{4}$ Department of Otorhinolaryngology, Head and Neck Surgery, Özel Esencan Hospital, Istanbul, Turkey
}

Objectives. To retrospectively evaluate the patients who underwent nasopharyngeal biopsy with imaging and biopsy results, who have or don't have symptoms for nasopharyngeal pathology and to determine the ratio of the nasopharyngeal cancer cases and other pathologic conditions.

Methods. In this retrospective study, 983 patients who underwent endoscopic nasopharyngeal biopsy for symptomatic nasopharyngeal lesions were included. All pathological results, benign or malign was recorded and classified due to the patients' presenting symptoms such as symptomatic for nasopharyngeal pathology or asymptomatic. Computed tomography (CT) or magnetic resonance imaging (MRI) reports were also recorded separately as group A for malignancy or group B for not malignancy.

Results. Forty-five (4.6\%) of 983 biopsies were malignant. In this group, there is no statistically significant difference between symptomatic and asymptomatic group. For malignant pathologies, the sensitivity of MRI was found $88.2 \%$ and CT was $61.5 \%$.

Conclusion. For early diagnosis of nasopharyngeal cancer, all patients admitted to Ear, Nose and Throat (ENT) referral clinics should be examined endoscopically irrespective of their complaints and suspicious cases should be investigated by imaging especially by MRI. If MRI report clearly indicates Thornwaldt cyst or reactive lymphoid hyperplasia and this result is compatible with endoscopic findings, biopsy may not be necessary. Apart from these cases, all suspected lesions should be biopsied.

Keywords. Nasopharyngeal Neoplasms; Biopsy; Multidetector Computed Tomography; Magnetic Resonance Imaging

\section{INTRODUCTION}

Nasopharyngeal carcinoma (NPC) is a unique disease with clinical behavior, epidemiology and histopathology that is different from that of the other squamous cell carcinomas of the head

- Received August 15, 2015

Revised April 1, 2016

Accepted June 5, 2016

- Corresponding author: Fatih Gul

Department of Otorhinolaryngology, Head and Neck Surgery, Bitlis Tatvan

State Hospital,Tatvan, Bitlis 13200, Turkey

Tel: +90-544-312-0607, Fax: +90-312-291-2786

E-mail: drfatihgul@gmail.com and neck [1,2]. This cancer is highly malignant with extensive infiltration, early lymphatic spread and notorious predilection for distant metastasis [3]. Despite growing incidence and awareness in the relevant populations, because of its silent, deep-seated location, majority of the patients already have advanced disease at the time of diagnosis $[3,4]$. NPC patients most commonly have a unilateral neck lump in $50 \%-70 \%$ of patients, from cervical lymph node metastasis; the tumor may not be clinically apparent at the time of presentation. Eustachian tube obstruction may produce persistent unilateral hearing loss or otitis media [2]. Other presenting symptoms include epistaxis, nasal obstruction or less frequently cranial nerve palsies [2-5].

Copyright (C) 2017 by Korean Society of Otorhinolaryngology-Head and Neck Surgery.

This is an open-access article distributed under the terms of the Creative Commons Attribution Non-Commercial License (http://creativecommons.org/licenses/by-nc/4.0)

which permits unrestricted non-commercial use, distribution, and reproduction in any medium, provided the original work is properly cited. 
Nasopharyngeal endoscopy is the initial investigation of choice for the detection of NPC with the definite diagnosis of NPC being confirmed by means of endoscopic biopsy of the site of the primary tumor [6]. Imaging modalities such as computed tomography (CT), magnetic resonance imaging (MRI) and positron emission tomography (PET) have been used for the diagnosis and staging of NPC $[5,6]$. The presence of high level anti-Epstein-BarrVirus (EBV) antibodies with NPC are well known. Serologic markers for EBV infection, such as antibodies to EBV immunoglobulin A or EBV DNA gene products have also been used for screening [7].

Despite all the advances in imaging and screening techniques, the current gold standard of clinical NPC detection is nasopharyngoscopy combined with biopsy of suspicious lesions.

Suspicion of NPC based on the presence of metastatic cervical lymph nodes or unilateral serous otitis media can be relatively easy. For early diagnosis improves survival and prognosis, the diagnosis of NPC patients before abovementioned marked symptoms emerge is important. Because of this, all patients admitted to reference center, should be examined endoscopically irrespective of their complaints and suspicious cases should be investigated by imaging and biopsy. In this research, we retrospectively investigated the patients who underwent nasopharyngeal biopsy with imaging and biopsy results, who have or don't have symptoms for NPC and determined the ratio of the NPC cases and other pathologic conditions with or without obvious symptoms.

\section{MATERIALS AND METHODS}

Nine hundred eighty-three patients (600 males and 383 females) aged from 10 to 85 years old (mean age, 39.7 years) who underwent endoscopic nasopharyngeal biopsy for suspicious nasopharyngeal lesion and whose required data were obtained from their files included in this retrospective research between 2010 and 2014 years, in Ankara Atatürk Training and Research Hospital ENT Clinic. Review of medical records was approved by the Institutional Review Board (IRB No: 2015186). All pathological results, benign or malign was recorded. Results were classified due to the patients presenting symptoms such as symptomatic group (343 patients) for nasopharyngeal pathology

\section{H I I G H L L I}

- Nearly half of the patients who were diagnosed malign nasopharyngeal pathology presented with asymptomatic.

- All patients should be examined endoscopically regardless of presenting symptoms.

- Magnetic resonance imaging was valuable for sensitivity when compared to endoscopic biopsy. or asymptomatic group (640 patients). Patients presenting with symptoms caused by the presence of a tumor mass in the nasopharynx (epistaxis, nasal obstruction, and discharge) symptoms associated with dysfunction of the Eustachian tube (hearing loss), symptoms associated with the superior extension of the tumor (headache, diplopia, facial pain, and numbness) and neck masses were included in the group of symptomatic for nasopharyngeal pathology and all other patients were included in the group of asymptomatic (diagnosed incidentally) detected by routine nasal endoscopy.

Amongst all patients who underwent nasopharyngeal biopsy we have obtained 765 of CT or MRI results (199 MRI and 566 CT). MRI was mainly used for looking to soft parts of nasopharynx and its around. But CT scan was performed in these conditions: (1) Incidental nasopharyngeal radiological finding on brain, paranasal or maxillofacial CT; (2) History of claustrophobia or placed cardiac pacemaker, vascular stent, artificial joints or any metallic piece; and (3) Being over table's weight limit. All imaging techniques were performed with or without intravenous contrast. If a patient had a history of severe asthma, chronic renal failure or allergic reactions due to contrast materials or unknown drugs, noncontrast imaging techniques were preferred. For each pathological diagnosis group, CT or MR imaging reports were recorded separately as group A for malignancy or group B for benign findings. Radiological findings for malignancy were; all asymmetric nasopharyngeal thickenings with or without contrast enhancement or invasion or destruction of surrounding tissues, and the cases reported as clearly malignant by the radiologist. All diffuse thickenings and reactive lymphoid hyperplasia and Thornwaldt cysts were recorded as group B.

All nasopharyngeal biopsies were performed under local anesthesia with tetracaine $4 \%$ and decongestion with oxymetazoline hydrochloride $0.05 \%$. A four-quadrant nasopharyngeal biopsy was taken using a punch forceps. The biopsy specimens were immersed in formalin and then embedded in paraffin. Sections cut from paraffin blocks were stained with hematoxylin and eosin. If there was a suspicious finding in an imaging technique, nasopharyngeal thickening was re-biopsied to confirm the result. Frozen biopsy was not performed in none of our patients.

All statistical analyses were performed by IBM SPSS ver. 22.0 (IBM Co., Armonk, NY, USA). Chi-square tests and Student $t$ test were performed to evaluate for statistical significance. A $P$ value of 0.05 or less was regarded as statistically significant.

\section{RESULTS}

Nine-hundred and eighty-three nasopharyngeal biopsies were performed during the study period. There were 383 females (39\%) and 600 males $(61 \%)$ whose median age at the time of the procedure was 39 years (range, 10 to 85 years). Forty-five 
(4.6\%) of the 983 pathologies were malignant. Twenty-nine (3\%) of the 45 malignant pathologies were NPC and remaining 17 (1.6\%) were other malignant pathologies.

Pathological reports of the benign and malign biopsies according to patients presenting symptoms and their distribution ratios are shown in Table 1. In malign pathologies group, there is no statistically significant difference between symptomatic and asymptomatic group $(P>0.05)$. However, there is a statistically significant difference between symptomatic and asymptomatic group in benign pathologies group $(P<0.001)$.

The results of CT or MR imaging reports for benign or malign lesions with each pathological diagnosis group and their suspicious for malignancy or not are shown in Table 2. For malignant pathologies the sensitivity of MRI was found $88.2 \%$, and CT was $61.5 \%$. For benign pathologies the specificity of MRI was found $98.8 \%$, and CT was $97.7 \%$. In CT groups; there is a stati-

Table 1. Distribution of pathological diagnosis associated with presenting symptoms

\begin{tabular}{lccccc}
\hline \multirow{2}{*}{ Pathological diagnosis } & $\begin{array}{c}\text { Symptomatic } \\
\text { group }\end{array}$ & & $\begin{array}{c}\text { Asymptomatic } \\
\text { group }\end{array}$ & P-value \\
\cline { 2 - 2 } & No. (\%) & & No. (\%) & \\
\hline Malign pathologies & $29(64.4)$ & & $16(35.6)$ & $<0.001$ \\
\hline Nasopharyngeal cancer & $18(40.0)$ & & $11(24.4)$ & \\
Lymphoma & $11(24.4)$ & & $4(8.9)$ & \\
\hline Plasmocitoma & - & & $1(2.2)$ & \\
Benign pathologies & $314(33.5)$ & & $624(66.5)$ & $<0.001$ \\
Reactive lymphoid hyperplasia & $293(31.2)$ & & $551(58.7)$ & \\
Thornwald cyst & $10(1.1)$ & & $50(5.3)$ & \\
Squamous metaplasia & $7(0.7)$ & & $10(1.1)$ & \\
Granulomatous inflammation & $3(0.3)$ & & $6(0.6)$ & \\
Actinomycosis & $1(0.1)$ & & $3(0.3)$ & \\
Hamartoma & - & & $3(0.3)$ & \\
Amyloidosis & - & & $1(0.1)$ & \\
\hline
\end{tabular}

cally significant difference between group A and B in benign pathologies $(P<0.001)$. However, there is no statically significant difference in malign pathologies $(P>0.05)$. In MRI groups; there are statically significant differences between group A and B in benign and malign pathologies ( $P<0.05$ in both groups). The most common finding for malignancy in both $\mathrm{CT}$ and MRI was asymmetric nasopharyngeal thickening. The most common clinical manifestation for malignancy was a nasopharyngeal mass $(16 / 45)$ in a routine endoscopic examination, followed by a cervical mass (15/45), serous otitis media (10/45) and epistaxis (4/45).

\section{DISCUSSION}

Though NPC is a rare disease on a world scale, and accounts for $0.7 \%$ of all cancers according to global cancer statistics, from the International Agency for Research on Cancer, there were over 84,000 new NPC cases in 2008 [8,9]. With evolution of treatment, this lethal cancer carries an excellent prognosis and maybe curable especially if detected early but most of the patients presented with stage III to IV disease with negatively affected the cure rate and increased the mortality rate $[3,7,10]$.

Imaging is an essential tool in the management of nasopharyngeal cancer. MRI is superior to $\mathrm{CT}$ in this regard because of the lack of artifact from dental fillings, the availability of highresolution coronal and sagittal imaging, and the exquisite lesion contrast relative to the brain, extracranial fat, and muscle. However, destruction of cortical bone (such as that of the skull base) is more easily visualized, and best evaluated by CT.Though MRI is currently the best technique to achieve an accurate diagnosis, CT may initially help to assess the nasopharyngeal lesion because it allows rapid image acquisition and is able to image bone, soft tissue and blood vessels all at the same time and costs

Table 2. Detailed imaging results of CT and MRI between group A (for malignant findings) and group B (for benign findings)

\begin{tabular}{|c|c|c|c|c|c|c|}
\hline \multirow{2}{*}{ Pathological diagnosis } & \multicolumn{2}{|c|}{ CT $(n=566)$} & \multirow{2}{*}{$P$-value } & \multicolumn{2}{|c|}{ MRI $(n=199)$} & \multirow{2}{*}{$P$-value } \\
\hline & Group A & Group B & & Group A & Group B & \\
\hline Malign pathologies & $16(61.5)$ & $10(38.5)$ & $>0.05$ & $15(88.2)$ & $2(11.8)$ & $<0.001$ \\
\hline Nasopharyngeal cancer & $11(42.3)$ & $2(7.7)$ & & $13(76.4)$ & $1(5.9)$ & \\
\hline Lymphoma & $5(19.2)$ & $8(30.8)$ & & $1(5.8)$ & $1(5.9)$ & \\
\hline Plasmocitoma & - & - & & $1(5.8)$ & 0 & \\
\hline Benign pathologies & $108(20.1)$ & $432(79.9)$ & $<0.001$ & $17(9.3)$ & $165(90.7)$ & $<0.001$ \\
\hline Reactive lymphoid hyperplasia & $99(18.3)$ & $392(72.6)$ & & $12(6.6)$ & $139(76.4)$ & \\
\hline Thornwaldt cyst & $4(0.7)$ & $27(5)$ & & 0 & $22(12.1)$ & \\
\hline Squamous metaplasia & $3(0.6)$ & $7(1.3)$ & & $2(1.1)$ & $2(1.1)$ & \\
\hline Granulomatous inflammation & $22(0.4)$ & $392(0.6)$ & & $1(0.5)$ & $1(0.5)$ & \\
\hline Actinomycosis & 0 & $27(0.4)$ & & $1(0.5)$ & 0 & \\
\hline Hamartoma & 0 & $1(0.2)$ & & $1(0.5)$ & $1(0.5)$ & \\
\hline Amyloidosis & - & - & & - & - & \\
\hline
\end{tabular}

Values are presented as number (\%).

$\mathrm{CT}$, computed tomography; MRI, magnetic resonance imaging. 
less than MRI (about half the price of MRI). In our study, 285 patients who were not convenient for MRI (patients with cardiac pacemakers, tattoos and metal implants, anxiety caused by claustrophobia and cost-effectiveness issues and getting faster results) were performed CT. In 314 patients, nasopharyngeal findings were incidentally found or discovered by other CTs (brain, maxillofacial, and paranasal).

Though serological screening programs for high-risk populations can be available, current gold standard of NPC detection is nasopharyngoscopy combined with biopsy of suspicious lesions $[4,11,12]$. Our research clearly showed that nearly half of the patients who were diagnosed malign nasopharyngeal pathology presented with asymptomatic. Therefore, especially in referral ENT centers, all patients should be examined endoscopically regardless of presenting symptoms. In the literature, the sensitivity of endoscopy for NPC has been reported nearly as $90 \%[5,6]$. This ratio may be true for the patients whose symptoms are highly suspicious for NPC. According to our research, this sensitivity rate is very high for the patients presenting with asymptomatic. In our research, malignant pathology was spotted in $4.6 \%$ of all of the specimens. In order to avoid unnecessary biopsies, imaging techniques (especially MRI) should be used for suspicious lesions. All minor bulging and asymmetries should be examined radiologically. Various radiological techniques can be used for the evaluation of nasopharyngeal lesions (CT, MRI, angiography and PET/CT) [13-16]. Amongst these, MRI is currently the best technique to achieve an accurate diagnosis. According to King et al. [6], nasopharyngeal MRI offers 95\% accuracy for the detection of primary NPC. In our research, MRI offers $88.2 \%$ sensitivity. This ratio was even lower for CT reports (61.5\%). Our low accuracy rate may be due to noncontrast MRI or CT scans performed in patients with a history of severe asthma, known allergy to contrast materials and renal disease. According to Liu et al. [13], malignant lymphoma is the second most common neoplasm of head and neck with Waldeyer's ring being the most common extra nodal site. Typically, nasopharyngeal non-Hodgkin's lymphoma is characterized as symmetrical and diffuse exophytic tumor that can extend into the nasopharyngeal airway rather than infiltrating deeply into the parapharyngeal region [13]. In contrast, NPC tends to be an asymmetrical tumor that has a propensity to undergo extensive invasion both laterally and in depth and so spread superiorly into the skull base, rather than inferiorly into the oropharynx [13]. Because of the diffuse and symmetrical nature of these tumors with noncontrast technique, our MRI and CT accuracy rate may be low. Moreover, it is hard to differentiate the NPC from the lymphoma on noncontrast radiological basis. On the other hand, in our research, the sensitivity of MR and CT imagings for Thornwaldt cysts were $100 \%$ and $87.1 \%$ respectively, and for reactive lymphoid hyperplasia were $92.1 \%$ and $79.9 \%$, respectively. In additionally, approximately, $5 \%$ of cancers were missed at endoscopic biopsy due to symmetric nasopharyngeal lesions or lack of deep biopsies; all of these cancers were identified at MR imaging. In this study, MRI was valuable for sensitivity when compared to endoscopic biopsy $(88.2 \%$ and $84.4 \%$, respectively). Biopsy has a disadvantage when taken a biopsy in a mistake region of nasopharynx which may be result as a reactive lymphoid hyperplasia.

In our study, the main limitation is that we did not compare the contrast and noncontrast technique. Noncontrast MRI for detection of low-grade nasopharyngeal cancers may be unnoticed when compared to contrast MRI.

In conclusion, MRI has the potential to be an initial diagnostic test for the detection of nasopharyngeal malignancy. MRI depicts subclinical cancers missed at endoscopy or endoscopic biopsy and identifies patients who do not have NPC and who therefore do not need to undergo invasive sampling biopsies and has an advantage in guiding biopsy in order to determine the site of the biopsy. Only the suspected lesions which are approved by MRI with contrast should be biopsied.

\section{CONFLICT OF INTEREST}

No potential conflict of interest relevant to this article was reported.

\section{REFERENCES}

1. Abdel Khalek Abdel Razek A, King A. MRI and CT of nasopharyngeal carcinoma. AJR Am J Roentgenol. 2012 Jan;198(1):11-8.

2. Goh J, Lim K. Imaging of nasopharyngeal carcinoma. Ann Acad Med Singapore. 2009 Sep;38(9):809-16.

3. Lee AW, Ng WT, Chan YH, Sze H, Chan C, Lam TH. The battle against nasopharyngeal cancer. Radiother Oncol. 2012 Sep;104(3): 272-8.

4. Ng RH, Ngan R, Wei WI, Gullane PJ, Phillips J. Trans-oral brush biopsies and quantitative PCR for EBV DNA detection and screening of nasopharyngeal carcinoma. Otolaryngol Head Neck Surg. 2014 Apr;150(4):602-9.

5. Gao Y, Liu JJ, Zhu SY, Yi X. The diagnostic accuracy of ultrasonography versus endoscopy for primary nasopharyngeal carcinoma. PLoS One. 2014 Mar;9(3):e90412.

6. King AD, Vlantis AC, Bhatia KS, Zee BC,Woo JK, Tse GM, et al. Primary nasopharyngeal carcinoma: diagnostic accuracy of MR imaging versus that of endoscopy and endoscopic biopsy. Radiology. 2011 Feb;258(2):531-7.

7. Romdhoni AC, Wiqoyah N, Kentjono WA. Early detection of nasopharyngeal carcinoma using IgA anti-EBNA1 + VCA-p18 serology assay. Ear Nose Throat J. 2014 Mar;93(3):112-5.

8. Song C, Yang S. A meta-analysis on the EBV DNA and VCA-IgA in diagnosis of Nasopharyngeal Carcinoma. Pak J Med Sci. 2013 May; 29(3):885-90.

9. Zhang L, Chen QY, Liu H, Tang LQ, Mai HQ. Emerging treatment options for nasopharyngeal carcinoma. Drug Des Devel Ther. 2013; 7:37-52.

10. Piazza C, Dessouky O, Peretti G, Cocco D, De Benedetto L, Nicolai P. Narrow-band imaging: a new tool for evaluation of head and neck 
squamous cell carcinomas: review of the literature. Acta Otorhinolaryngol Ital. 2008 Apr;28(2):49-54.

11. Xiao L, Xiao T, Wang ZM, Cho WC, Xiao ZQ. Biomarker discovery of nasopharyngeal carcinoma by proteomics. Expert Rev Proteomics. 2014 Apr;11(2):215-25.

12. Stevens SJ, Verkuijlen SA, Hariwiyanto B, Harijadi, Paramita DK, Fachiroh J, et al. Noninvasive diagnosis of nasopharyngeal carcinoma: nasopharyngeal brushings reveal high Epstein-Barr virus DNA load and carcinoma-specific viral BARF1 mRNA. Int J Cancer. 2006 Aug;119(3):608-14.

13. Liu XW, Xie CM, MoYX, Zhang R, Li H, Huang ZL, et al. Magnetic resonance imaging features of nasopharyngeal carcinoma and nasopharyngeal non-Hodgkin's lymphoma: are there differences? Eur J Radiol. 2012 Jun;81(6):1146-54.

14. Weber AL. Computed tomography and magnetic resonance imaging of the nasopharynx. Isr J Med Sci. 1992 Mar-Apr;28(3-4):161-8.

15. Kosling S, Knipping S, Hofmockel T. Imaging of nasopharyngeal diseases. HNO. 2009 Aug;57(8):813-24.

16. Cho KS, Kang DW, Kim HJ, Lee JK, Roh HJ. Differential diagnosis of primary nasopharyngeal lymphoma and nasopharyngeal carcinoma focusing on CT, MRI, and PET/CT. Otolaryngol Head Neck Surg. 2012 Apr;146(4):574-8. 